\title{
INCORPORAÇÃO DE CÉRIO À PENEIRA MOLECULAR MESOPOROSA (MCM-41)
}

\author{
G. E. do NASCIMENTO, M. M. M. B. DUARTE e C. M. B. M. BARBOSA \\ Universidade Federal de Pernambuco, Departamento de Engenharia Química \\ E-mail para contato: grazielen@yahoo.com.br
}

\begin{abstract}
RESUMO - A síntese e caracterização da peneira molecular mesoporosa MCM-41 vêm sendo amplamente estudadas devido a sua elevada área superficial e volume de poros de grandes dimensões, visando sua potencial aplicação em processos de adsorção, separação e catálise. Neste estudo, a peneira molecular mesoporosa com incorporação do metal de transição Cério (Ce-MCM-41) foi sintetizada pelo método hidrotérmico. A composição molar do gel obtido foi de 1 CTMABr: $4 \mathrm{SiO}_{2}$ : $1 \mathrm{Na}_{2} \mathrm{O}: 0,2 \mathrm{Ce}_{2} \mathrm{O}_{3}: 200 \mathrm{H}_{2} \mathrm{O}$. A caracterização do material foi realizada por TG, DRX, BET e FT-IR. O material apresentou boa organização estrutural e morfológica segundo as analises de DRX e BET. Os espectros de FT-IR apresentaram bandas na região de 400 a $4000 \mathrm{~cm}^{-1}$ e juntamente com a análise de TG revelaram que a remoção do direcionador estrutural foi efetiva. Diante do exposto, a incorporação do metal cério não afetou a estrutura da MCM-41 e o método de síntese empregado foi eficiente uma vez que houve formação de material mesoporoso.
\end{abstract}

\section{INTRODUÇÃO}

A peneira molecular mesoporosa MCM-41, pertencente a família M41S, foi primeiramente descoberta pela Mobil Company em 1992 (Beck et al., 1992). Desde então, grande interesse tem sido focado em suas propriedades e aplicações, devido às suas elevadas áreas superficiais específicas, distribuição de tamanho de poros estreitos e controláveis e um amplo espectro de diâmetros de poro (15-100Å) na gama mesoporosa (Jiang et al., 2007; Zhao et al., 2011).

As peneiras moleculares mesoporosas podem ser aplicadas nas áreas de catálise, adsorção e controle da poluição do meio ambiente (Hao et al., 2006; Qin et al, 2007; Puanngama e Unob, 2008).

No entanto, a base da peneira molecular mesoporosa é sílica amorfa e contém uma quantidade considerável de grupos silanóis. A fim de ampliar o campo de sua aplicação há necessidade de melhorias na síntese com a substituição do silício por outros íons. A incorporação de heteroátomos às paredes da MCM-41 permite o controle de suas características, viabilizando a obtenção de materiais com propriedades pré-estabelecidas (Oliveira et al., 2005).

As peneiras moleculares podem gerar sítios mais básicos ou ácidos conforme aplicação desejada (Li et al, 2010). Nas últimas décadas, a incorporação de heteroátomos à estrutura da MCM-41 tem 


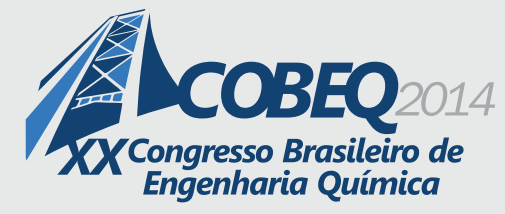

sido amplamente investigada (González Vargas et al., 2013; Li et al., 2013).

A incorporação de metais de transição (Nilsen et al., 2007; Anunziata et al., 2008), metais de terras raras (Zhao et al., 2011; Akondi et al., 2012) e metais alcalinos terrosos (Wu et al., 2011) à MCM-41 produz novos tipos de adsorventes utilizados em diversas aplicações como para remoção de contaminantes de derivados de petróleo.

Desta forma, este trabalho teve como objetivo o estudo da incorporação do cério à peneira molecular mesoporosa MCM-41 através de sua síntese e caracterização.

\section{EXPERIMENTAL}

\subsection{Síntese da Peneira Molecular Mesoporosa Ce-MCM-41}

A peneira molecular mesoporosa Ce-MCM-41 foi sintetizada pelo método hidrotérmico (Nascimento, 2011), onde foram utilizados sílica gel e silicato de sódio como fonte de silício, brometo de cetiltrimetilamônio (CTMABr) como direcionador estrutural, nitrato de cério como fonte de metal de transição e água destilada. A síntese foi conduzida à $100^{\circ} \mathrm{C}$ em autoclaves de teflon encamisadas em aço inox por um período de cinco dias com correção diária de $\mathrm{pH}$ na faixa de 9,5-10,0 com uma solução $30 \%$ de ácido acético. A composição molar do gel obtido foi de: 1 CTMABr: $4 \mathrm{SiO}_{2}: 1 \mathrm{Na}_{2} \mathrm{O}$ : $0,2 \mathrm{Ce}_{2} \mathrm{O}_{3}: 200 \mathrm{H}_{2} \mathrm{O}$. Em seguida, o material obtido foi submetido a um processo de calcinação utilizando uma rampa de aquecimento de $5^{\circ} \mathrm{C} \cdot \mathrm{min}^{-1}$, partindo da temperatura ambiente até $500^{\circ} \mathrm{C}$ em atmosfera inerte de nitrogênio a uma vazão de $100 \mathrm{~mL} \cdot \mathrm{min}^{-1}$ permanecendo nesta condição por 1 hora. Em seguida, o fluxo foi trocado por ar sintético na vazão de $100 \mathrm{~mL} . \mathrm{min}^{-1}$ por tempo adicional de 1 hora.

\subsection{Caracterização da Peneira Molecular Mesoporosa Ce-MCM-41}

A caracterização do material foi realizada por Análise térmica (TG); Difração de raios-X (DRX); Adsorção/dessorção de $\mathrm{N}_{2}$ por BET e Método BJH e Espectroscopia na região do Infravermelho com Transformada de Fourier (FT-IR).

A curva termogravimétrica foi obtida em uma termobalança NETZSCH modelo STA 449 F3 Jupiter, em atmosfera de $100 \mathrm{~mL} \cdot \mathrm{min}^{-1}$ de nitrogênio. As medidas de difração de raios X foram realizadas em um difratômetro BRUKER D8 ADVANCE, usando radiação $\mathrm{Cu}-\mathrm{K} \alpha$. As isotermas de adsorção/dessorção de $\mathrm{N}_{2}$ foram obtidas pelo equipamento ASAP 2420 da MICROMERITICS. Os espectros de absorção na região do infravermelho foram obtidos em um espectrofotômetro de absorção no infravermelho VERTEX 70 da BRUKER, usando KBr como agente dispersante.

\section{RESULTADOS E DISCUSSÃO}

\subsection{Caracterização da Peneira Molecular Mesoporosa Ce-MCM-41}

Análise térmica (TG): A curva da análise termogravimétrica (TG) para o Ce-MCM-41 está 
apresentada na Figura 1.

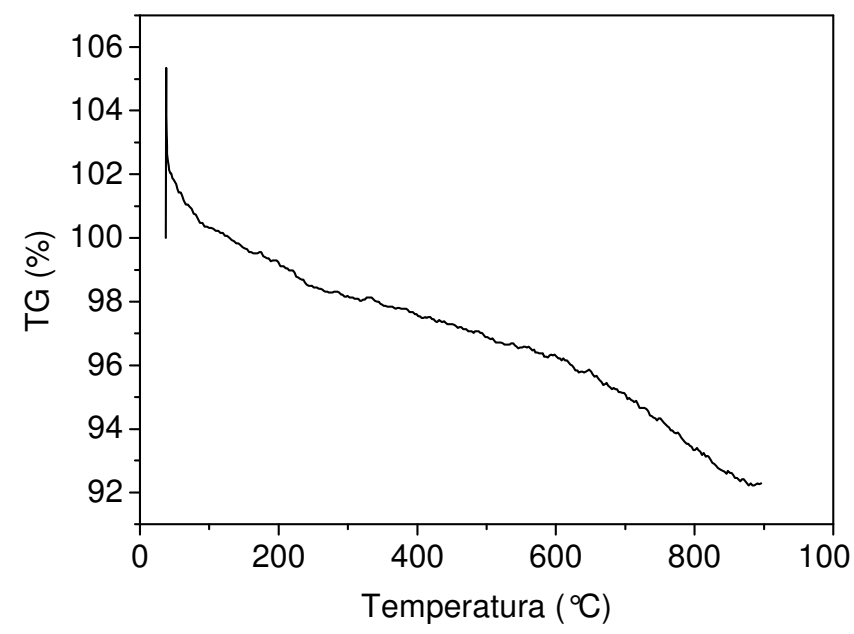

Figura 1 - Curva termogravimétrica para o material sintetizado.

Verifica-se através da curva TG para o adsorvente Ce-MCM-41 três perdas de massa. A primeira perda de massa foi de 5,0\%, sendo atribuída a dessorção de água fisicamente adsorvida. A segunda perda de massa foi na ordem de 3,0\%, devido à decomposição do surfactante ocluído nos poros do adsorvente. Após a decomposição do surfactante, verifica-se a terceira perda de massa de $4,0 \%$, resultante da condensação dos grupos silanóis da superfície interna dos poros que interagiam com as moléculas do direcionador. Estes resultados estão de acordo com a literatura (Park et al., 2007; Ghorbani et al., 2013; Nascimento et al., 2014).

Difração de Raios-X (DRX): Os difratogramas obtidos para o material, antes e após calcinação estão apresentados na Figura 2.

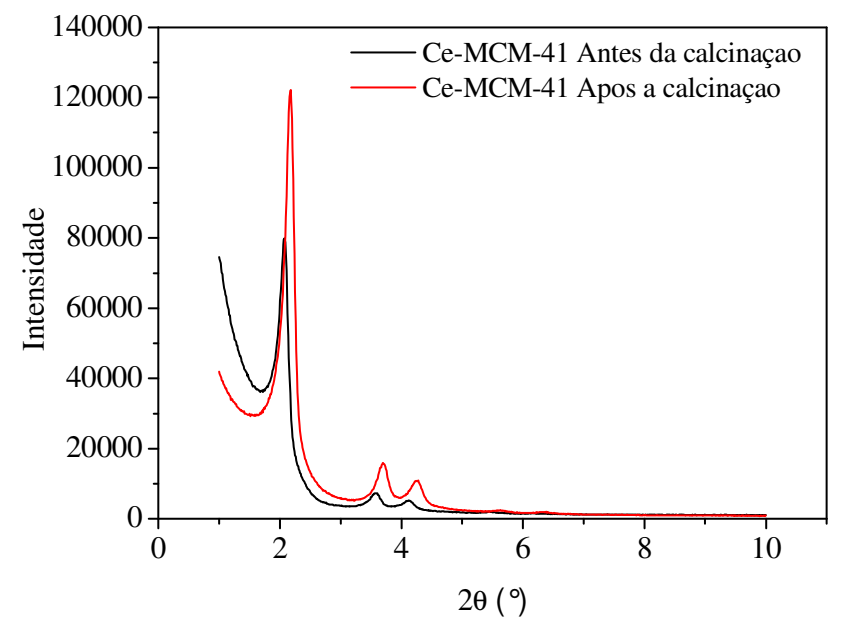

Figura 2 - Difratograma de raios-X antes e após a calcinação. 


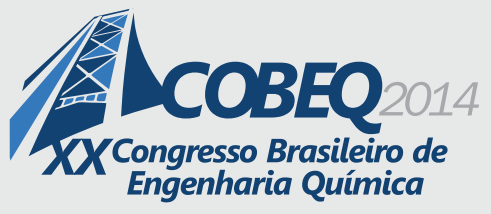

O material sintetizado apresentou padrão de raios-X similar com o apresentado na literatura para materiais mesoporosos (Beck et al, 1992; Chien et al., 2005; Du e Yang, 2012; Ghorbani et al., 2013; Nascimento et al., 2014). Verifica-se a presença dos planos (100), (110) e (200), compatível com padrão da MCM-41. Após a calcinação verificou-se um aumento nas intensidades dos picos o que sugere um grau de organização da amostra, comprovando que a remoção do direcionador não afetou a estrutura da Ce-MCM-41.

Os resultados dos parâmetros cristalográficos via difração de raios-X para o Ce-MCM-41 antes e após a calcinação podem ser verificados na Tabela 1.

Tabela 1 - Resultados da análise de difração de raios-X.

\begin{tabular}{|c|c|c|}
\hline Propriedades & Antes da calcinação & Após a calcinação \\
\hline $2 \theta$ & 2,11 & 2,17 \\
\hline $\mathrm{d}(\mathrm{nm})$ & 4,19 & 4,07 \\
\hline $\mathrm{a}_{0}(\mathrm{~nm})$ & 4,84 & 4,70 \\
\hline
\end{tabular}

O material sintetizado apresentou um pico em $2 \theta=2,11$ e dimensão do parâmetro de rede hexagonal $\left(\mathrm{a}_{0}\right)$ de $4,84 \mathrm{~nm}$, conforme a Lei de Bragg. Após a calcinação o Ce-MCM-41 apresentou $\mathrm{a}_{0}=4,70 \mathrm{~nm}$, o que significa que a remoção do direcionador CTMABr promoveu contração da rede cristalina, mas não acompanhada de diminuição do ordenamento estrutural, uma vez que a intensidade do pico (100) aumentou, como pode ser observado através da Figura 2.

Adsorção/Dessorção de $\mathrm{N}_{2}$ por BET e Método BJH: As isotermas de adsorção/dessorção de $\mathrm{N}_{2}$ para o Ce-MCM-41 após a calcinação estão apresentadas na Figura 3.

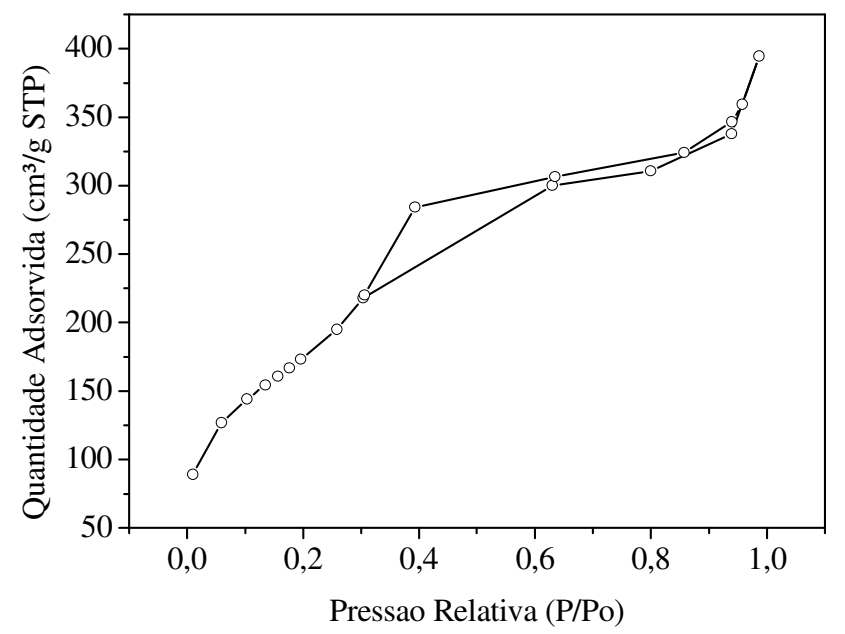

Figura 3 - Isoterma de adsorção/dessorção de $\mathrm{N}_{2}$ após a calcinação.

Observa-se na Figura 3 que as isotermas são do tipo IV conforme a International Union of Pure 
and Applied Chemistry (IUPAC), características de materiais mesoporosos (IUPAC, 1982). Tabela 2 .

A caracterização textural do adsorvente obtida pelo método BET e BJH pode ser verificada na

Tabela 2 - Caracterização textural do Ce-MCM-41 obtida pelos métodos BET e BJH.

\begin{tabular}{|c|c|c|}
\hline Características & Antes da calcinação & Após a calcinação \\
\hline $\begin{array}{c}\text { Área superficial BET } \\
{\left[\mathrm{m}^{2} \cdot \mathrm{g}^{-1}\right]}\end{array}$ & 395,4 & 756,8 \\
\hline $\begin{array}{c}\text { Volume de poros BJH } \\
{\left[\mathrm{cm}^{3} \cdot \mathrm{g}^{-1}\right]}\end{array}$ & 0,37 & 0,76 \\
\hline $\begin{array}{c}\text { Tamanho dos poros BJH } \\
{[\AA]}\end{array}$ & 41,7 & 44,3 \\
\hline
\end{tabular}

O material obtido após a calcinação apresentou tamanho de poros de 44,3 $\AA$, que de acordo com a IUPAC são classificados como materiais mesoporosos confirmando o observado na Figura 3.

O Ce-MCM-41 apresentou maior área superficial após a calcinação, como era esperado pela análise dos difratogramas, uma vez que após este processo o material apresentou maior intensidade do pico característico (100). Estes resultados estão de acordo com a literatura (Beck et al., 1992; Chien et al., 2005; Du e Yang, 2012; Jiang et al., 2012; Ghorbani et al., 2013; Nascimento et al., 2014).

Espectroscopia na região do Infravermelho com Transformada de Fourier: Os espectros de infravermelho do Ce-MCM-41, antes e após calcinação estão apresentados na Figura 4.

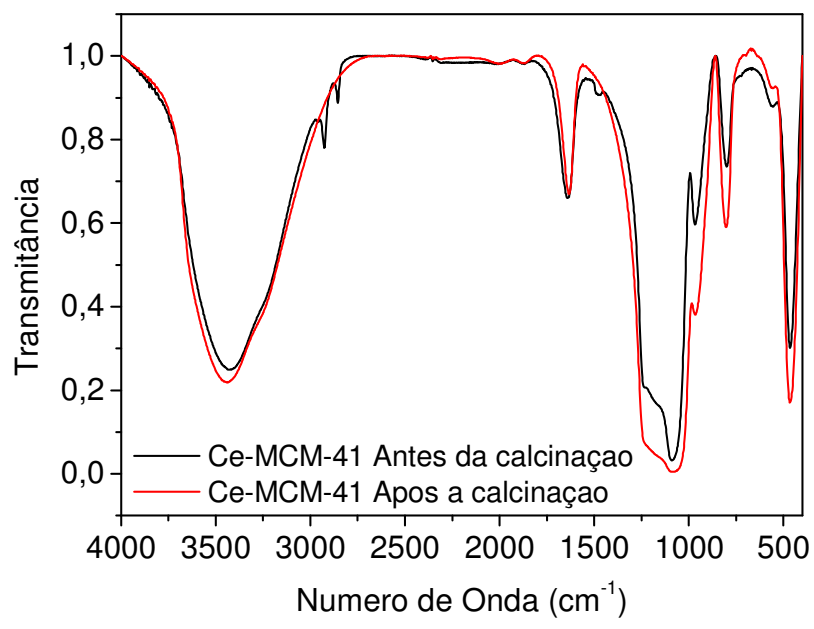

Figura 4 - Espectros de FT-IR antes e após a calcinação.

Os espectros de absorção no infravermelho das amostras de Ce-MCM-41 antes e após a calcinação apresentam bandas na região de 400 a $4000 \mathrm{~cm}^{-1}$, características das vibrações 


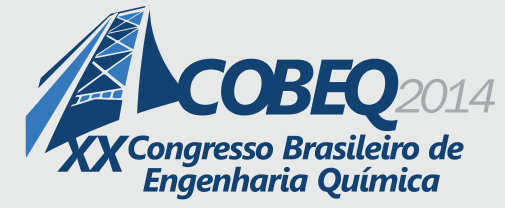

19 a 22 de outubro de 2014

Florianópolis/SC

fundamentais da rede da MCM-41, em concordância com a literatura (Silva, 2009).

Na região de 1000 a $1250 \mathrm{~cm}^{-1}$ há uma banda referente ao estiramento assimétrico de Si-O-Si. A banda em torno de $800 \mathrm{~cm}^{-1}$ refere-se ao estiramento simétrico da ligação Si-O-Si na rede. O espectro apresenta ainda uma banda de baixa densidade a $970 \mathrm{~cm}^{-1}$, que é atribuída ao estiramento da ligação $\mathrm{Si}-\mathrm{OH}$ e Si-OM $(\mathrm{M}=$ metal$)$, indicando a presença do cério na estrutura. Esse espectro também mostra uma banda de absorção larga em $3400 \mathrm{~cm}^{-1}$, correspondente ao estiramento da ligação O-H da água e outra banda em $1635 \mathrm{~cm}^{-1}$ referente à água adsorvida na superfície.

A presença do direcionador pode ser confirmada pelas bandas na região de 2820 a $2950 \mathrm{~cm}^{-1}$ referentes aos estiramentos C-H. Após a calcinação, estas bandas relativas ao agente direcionador desapareceram, indicando que o processo de calcinação foi eficiente na remoção desse composto. Esses resultados corroboram com os encontrados na análise termogravimétrica quando se verificou a perda de massa devido à remoção do agente direcionador de estrutura.

Estes resultados estão de acordo com os encontrados por Silva (2009); Du e Yang (2012); Ghorbani et al., (2013) e Nascimento et al. (2014).

\section{CONCLUSÃO}

O método de síntese empregado para a obtenção do adsorvente Ce-MCM-41 foi eficiente uma vez que houve formação de material mesoporoso e a incorporação do metal cério não afetou a estrutura da MCM-41 conforme os resultados obtidos na caracterização do material. Com base no exposto, foi obtido um material do tipo MCM-41 visando futuras aplicações em processos adsortivos para remoção de contaminantes na indústria de petróleo.

\section{AGRADECIMENTOS}

PRH-28, ANP, PETROBRÁS, UFPE e ao CETENE pelas análises de caracterização do material.

\section{REFERÊNCIAS}

AKONDI, A. M.; TRIVEDIA, R.; SREEDHARA, B.; KANTAM, M. L.; BHARGAVA, S. Ceriumcontaining MCM-41 catalyst for selective oxidative arene cross-dehydrogenative coupling reactions. Catal. Today, v. 198, 35-44, 2012.

ANUNZIATA, O. A.; BELTRAMONE, A. R.; CUSSA, J. Synthesis at atmospheric pressure and characterization of highly ordered Al, V, and Ti-MCM-41 mesostructured catalysts. Catal. Today, v. 133-135, p. 891-896, 2008.

BECK, J.S; VARTULI J. C.; ROTH W. J.; LEONOWICZ M. E.; KRESGE C. T.; SCHMITT K. D. A new family of mesoporous molecular sieves prepared with liquid cristal templates. J. Am. Chem. Soc., v. 114, p. 10834-10843, 1992.

CHIEN, S.; KUO, M.; CHEN, C. Synthesis, Characterization and Catalysis of Ce-MCM-41. J. Chin. 


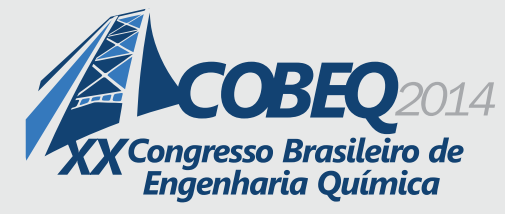

Chem. Soc., v. 52, p. 733-740, 2005.

DU, C.; YANG, H. Investigation of the physicochemical aspects from natural kaolin to Al-MCM-41 mesoporous materials. J. Colloid Interface Sci., v. 369, p. 216-222, 2012.

GHORBANI, F; YOUNESI, H.; MEHRABAN, Z.; ÇELIK, M. S.; GHOREYSHI, A. A.; ANBIA, M. Preparation and characterization of highly pure silica from sedge as agricultural waste and its utilization in the synthesis of mesoporous silica MCM-41. J. Taiwan Inst. Chem. E., v. 44, p. 821828, 2013.

GONZÁLEZ VARGAS, O.A.; DE LOS REYES HEREDIA, J.A.; MONTESINOS CASTELLANOS, A.; CHEN, L.F.; WANG. J.A. Cerium incorporating into MCM-41 mesoporous materials for CO oxidation. Mater. Chem. Phys., v. 139, p. 125-133, 2013.

HAO, X.-Y.; ZHANG, Y.-Q.; WANG, J.-W.; ZHOU, W.; ZHANG, C.; LIU, S. A novel approach to prepare MCM-41 supported $\mathrm{CuO}$ catalyst with high metal loading and dispersion. Microporous Mesoporous Mater., v. 88, p. 38-47, 2006.

International Union of Pure and Applied Chemistry (IUPAC). Reporting Physisorption data for gas/solid systems.v. 54, p. 2201-2218, 1982.

JIANG, C.; SU, A.; LI, X.; ZHOU, T.; HE, D. Study on the hydrothermal stability of MCM-41 via secondary restructure. Powder Technol., v. 221, p. 371-374, 2012.

JIANG, T.S.; ZHAO, Q.; YIN, H.B. Synthesis of highly stabilized mesoporous molecular sieves using natural clay as raw material. Appl. Clay Sci., v. 35, p. 155-161, 2007.

LI, D.; MIN, H.; JIANG, X.; RAN, X.; ZOU, L.; FAN, J. One-pot synthesis of Aluminum-containing ordered mesoporous silica MCM-41 using coal fly ash for phosphate adsorption. J. Colloid Interface Sci., v.404, p.42-48, 2013.

LI, X.; LI, B.; XU, J.; WANG, Q.; PANG, X.; GAO, X.; ZHOU, Z.; PIAO, J. Synthesis and characterization of Ln-ZSM-5/MCM-41 ( $\mathrm{Ln}=\mathrm{La}, \mathrm{Ce})$ by using kaolin as raw material. Appl. Clay Sci., v. 50 , p. $81-86,2010$.

NASCIMENTO, G. E.; DUARTE, M. M. M. B.; SCHULER, A. R. P.; BARBOSA, C. M. B. M. Synthesis, characterization, and application of the mesoporous molecular sieve Sr-MCM-41 in the removal of naphthenic acids from a model mixture of aviation kerosene by adsorption. Brazilian Journal of Petroleum and Gas. v. 8, n. 1, p. 001-013, 2014.

NILSEN, M. H.; ANTONAKOU, E.; BOUZGA, A.; LAPPAS, A.; MATHISEN, K.; STOCKER, M. Investigation of the effect of metal sites in Me-Al-MCM-41 $(\mathrm{Me}=\mathrm{Fe}, \mathrm{Cu}$ or $\mathrm{Zn})$ on the catalytic behavior during the pyrolysis of wooden based biomass. Microporous Mesoporous Mater., v. 105, p. 189-203, 2007.

OLIVEIRA, A. C.; RANGE, M. C.; FIERRO, J. L. G.; REYES, P.; OPORTUS, M. Efeito do Cromo nas Propriedades Catalíticas da MCM-41. Quim. Nova, v. 28, p. 37-41, 2005.

PARK, S. H.; KIM, B. H.; SELVARAJ, M.; LEE, T. G. Synthesis and Characterization of Mesoporous Ce-Mn-MCM-41 Molecular Sieves. J. Ind. Eng. Chem., v. 13, p. 637-643, 2007. 
PUANNGAMA, M., UNOB, F. Preparation and use of chemically modified MCM-41 and silica gel as selective adsorbents for $\mathrm{Hg}$ (II) ions. J. Hazard. Mater., v. 154, p. 578-587, 2008.

QIN, Q., MA, J., LIU, K. Adsorption of nitrobenzene from aqueous solution by MCM-41. J. Colloid Interface Sci., v. 315, p. 80-86, 2007.

SILVA, M. L. S. Síntese e Caracterização de Peneiras Moleculares Mesoporosas do tipo MCM-41 e AlMCM-41 a partir de fontes alternativas de sílica e de alumínio. 82F. Tese de Doutorado. Programa de Pós-Graduação em Ciência e Engenharia de Materiais da Universidade Federal do Rio Grande do Norte, 2009.

WU, G.; JIANG, S.; LI, L.; GUAN, N. Nitridation of $\mathrm{BaO}$ supported on mesoporous materials: Basicity characterization and catalytic properties. Appl. Catal., A., v. 391, p. 225-233, 2011.

ZHAO, Q., WANG, G.W., WU, D.L., ZHOU, X.P., JIANG, T.S. Microwave synthesis and textural property of europium substituted mesoporous molecular sieves. J. Phys. Chem. Solids., v. 72, p. 34$38,2011$. 